\title{
Association of lncRNA PVT1 Gene Polymorphisms with the Risk of Essential Hypertension in Chinese Population
}

\author{
Rong Li, ${ }^{1}$ Xia Yu, ${ }^{1}$ Yang Chen, ${ }^{1}$ Mulun Xiao, ${ }^{2}$ Meiling Zuo, ${ }^{3}$ Yuanlin Xie, ${ }^{3}$ \\ Zhousheng Yang $\left(\mathbb{1}^{4}\right.$, and Dabin Kuang $\mathbb{1}^{3}$ \\ ${ }^{1}$ Institute of Pharmacy \& Pharmacology and the Second Affiliated Hospital, University of South China, Hengyang 421001, China \\ ${ }^{2}$ Department of Urology, The First Affiliated Hospital of Zhengzhou University, Zhengzhou 450000, China \\ ${ }^{3}$ The Affiliated Changsha Hospital of Hunan Normal University, Changsha, Hunan 410006, China \\ ${ }^{4}$ Department of Pharmacy, The People's Hospital of Guangxi Zhuang Autonomous Region, Nanning, China
}

Correspondence should be addressed to Dabin Kuang; kdb@hunnu.edu.cn

Received 15 March 2021; Accepted 18 December 2021; Published 6 January 2022

Academic Editor: Jing $\mathrm{He}$

Copyright (C) 2022 Rong Li et al. This is an open access article distributed under the Creative Commons Attribution License, which permits unrestricted use, distribution, and reproduction in any medium, provided the original work is properly cited.

Vascular dysfunction and hyperlipidemia are essential risk factors contributing to essential hypertension (EH). The plasmacytoma variant translocation 1 (PVT1) is involved in modulating angiogenesis in tumor tissues and plays an important role in fat differentiation in the progress of obesity. Therefore, we selected two tagSNPs of PVT1 (rs10956390 and rs80177647) to investigate whether they are contributing to the risk of hypertension in Chinese patients. In total, 524 adult patients with $\mathrm{EH}$ and 439 matched healthy controls were enrolled for two central of China. Results. PVT1 rs10956390 and rs80177647 polymorphisms were genotyped by using TaqMan assay. PVT1 rs10956390 TT genotype was associated with a decreased risk of $\mathrm{EH}(\mathrm{OR}=0.561,95 \% \mathrm{CI}=0.372-0.846, P=0.006)$, while $\mathrm{rs} 80177647$ TA genotype was associated with an increased risk $(\mathrm{OR}=2.236,95 \% \mathrm{CI}=1.515-3.301, P<0.001)$. Rs $10956390 \mathrm{~T}$ allele was associated with lower triglyceride levels in the plasma both from healthy and EH donors. What is more, there is an association between rs 10956390 polymorphism and HDL-C level, as well as LDL-C. Conclusion. PVT1 rs10956390 and rs80177647 polymorphisms may contribute to the risk of EH in Chinese population by regulating blood lipid levels.

\section{Introduction}

Essential hypertension (EH), a noninfectious multifactorial disease, is a crucial risk factor for the morbidity and mortality of cardiovascular disease worldwide [1]. For the low treatment rate, control rate, and poor prognosis in $\mathrm{EH}$ patients, there is an urgent need to reveal the complex mechanism of EH. Over decades, accumulating evidence has confirmed that the factors causing hypertension include genegene and gene-environment interactions as well as biological systems [2].

Long noncoding RNAs (lncRNAs) are a class of noncoding RNAs longer than $200 \mathrm{nt}$. After long-time defined as "gene desert" for a long time, it has been recognized that lncRNAs play a pivotal role in diseases including cardiovascular diseases in a delicate and sophisticated network modulation manner
[3-6]. The plasmacytoma variant translocation 1 (PVT1) is located at cancer risk region 8q24.21 [7], which has been identified to aberrantly expressed in various cancers including pancreatic [8], prostate $[9,10]$, bladder [11] cancers, and hepatocellular carcinoma [12]. Zhao et al. found that PVT1 activates STAT3/VEGFA signaling axis to boost angiogenesis in gastric cancer [13]. Similarly, Zheng et al. revealed that PVT1 orchestrates the angiogenesis of vascular endothelial cells by evoking connective tissue growth factor (CTGF) and angiopoietin 2 (ANGPT2) expression in a miR-26b dependent manner [14]. Sun et al. found that PVT1 reduces the expression of miR-190a-5p in vascular endothelial cells (ECs), resulting in proliferation [15]. Guo et al. unraveled that PVT1 knockdown ameliorates ox-LDL-induced vascular endothelial cell injury and atherosclerosis through the miR-153-3p/GRB2 axis [16]. Furthermore, recent studies have shown that PVT1 
was found to be a potential biomarker for obesity treatment [17]. The potential mechanism in the interaction between obesity, atherosclerosis, and hypertension is elaborated and sophisticated, which contains activation of the sympathetic nervous system, epithelial dysfunction and oxidative stress, leptin, and adiponectin [18]. In the light of all the above, we assume that PVT1 may regulate biological processes of angiogenesis abnormality-associated diseases including diabetes, obesity, and hypertension. However, it is not clear whether PVT1 can regulate hypertension by affecting the blood lipid levels in the human plasma.

Accumulating evidence has shown that genetic polymorphism may be a novel treatment strategy to improve the control and management of diseases. Notably, in a recent study, Yan et al. revealed that PVT1 rs4410871 was a protective factor for coronary heart disease (CHD) susceptibility in Chinese population and influenced the complications (hypertension or diabetes) [19]. Considering the key role of PVT1 in affecting angiogenesis and regulating obesity, PVT1 may be a potential candidate gene related to $\mathrm{EH}$ risk. However, there is no report on the correlation between PVT1 polymorphism and EH risk. To clarify the clinical relevance of PVT1 polymorphisms, we conducted a case-control study in this article to investigate the association between PVT1 polymorphisms and the risk of $\mathrm{EH}$ in Chinese population. Meanwhile, we are trying to provide a promising biomarker in the subsequent occurrence mechanism of $\mathrm{EH}$ and improve the prognosis for patients.

\section{Materials and Methods}

2.1. Subjects. $261 \mathrm{EH}$ patients and 294 healthy controls were consecutively recruited between January 2021 and March 2021 from the second affiliated hospital of south China. 263 $\mathrm{EH}$ patients and 145 control individuals were enrolled between January 2018 and December 2019 from the First People's Hospital of Jining City, Shandong Province, in the north of China. The information of all participants including gender, age, BMI, smoking history, blood pressure, triglyceride (TG), total cholesterol (TC), high-density lipoprotein (HDL), lowdensity lipoprotein (LDL), and blood glucose was obtained. The presence of hypertension was clinically defined as having a systolic blood pressure (SBP) of at least $140 \mathrm{mmHg}$ and a diastolic blood pressure (DBP) of at least $90 \mathrm{mmHg}$ (without any antihypertensive medication), 30 years $\leq$ age $\leq 70$ years, and the course of hypertension between 1 year and 15 years. The exclusion criteria were as follows: severe organic lesions, with other malignancies, secondary hypertension, and recent history of glucocorticoid use. The healthy subjects undergoing routine healthy examinations were enrolled in the same period. This study protocol was approved by the Medical Ethics Committee of hospital involved in the study, and the written informed consent was gained from all participants or their first-degree relatives.

2.2. Genomic DNA Extraction and Genotyping. EDTA anticoagulation tubes were used to collect the peripheral blood samples of the subjects and stored at $-20^{\circ} \mathrm{C}$ until analysis. Use the E.Z.N.A.TM Blood DNA Midi kit (D3494, Omega) to extract and purify the genomic DNA following the standard protocol of the kit.

Genetic polymorphisms were screened by the 1000 Genomes Project (http://www.internationalgenome.org/). Haploview 4.2 was used to select according to $\mathrm{CHB}$ and $\mathrm{CHS}$, and the minimum allele frequency (Minor Allele Frequency (MAF)) was greater than 5\%. At last, we selected two PVT1 tagSNPs (rs10956390 and rs80177647).

Genomic DNA was diluted to working concentrations of $20 \mathrm{ng} / \mathrm{L}$ for genotyping. The assay was performed utilizing $\operatorname{TaqMan}^{\mathrm{TM}}$ Genotyping Master Mix (4371355, Thermo) as recommended by the manufacturer. Assay ID for rs10956390 is C_317048_10 (Thermo), and assay ID for rs80177647 is C_1101092625_10 (Thermo). SNP genotyping was performed by TaqMan real-time PCR system as reported elsewhere. And the reaction was conducted under the following conditions: $95^{\circ} \mathrm{C}$ for $10 \mathrm{~min}$, followed by 45 two-step cycles of $95^{\circ} \mathrm{C}$ for $15 \mathrm{~s}$ and $60^{\circ} \mathrm{C}$ for $1 \mathrm{~min}$.

2.3. Statistical Assay. Statistical analysis was performed using SPSS 19.0 (version 19.0 for Windows; Chicago, IL, USA). Continuous variables were presented as mean \pm standard deviation (SD) or the mean \pm standard error of the mean (SEM). Student $t$-tests were conducted to analyze the differences between two groups, while the significance of differences among multiple groups was evaluated using ANOVA. Hardy-Weinberg Equilibrium (HWE) was done by using $\chi^{2}$ test to validate the genotype frequency. Logistic regression was used to determine the association between PVT1 polymorphism and the risk of EH adjusting for multiple EH risk factors, such as the smoking history and blood glucose. In genetic association analysis, we used additive, dominant, and recessive genetic models. A two-tailed $P$ value $<0.05$ was considered as statistically significant.

\section{Results}

3.1. The Baseline Characteristics of the Subjects. The demographic and clinical characteristics of EH and control subjects were shown in Table 1. A total of 963 subjects ( 535 males, 428 females), with a mean age of $53 \pm 8$ years old, were selected including $524 \mathrm{EH}$ patients and 439 control subjects. In cases, there are 261 and $263 \mathrm{EH}$ patients from the south and north of China, respectively. The distribution of smoking history and the mean of body mass index (BMI, $P<0.001$ ), fasting blood glucose (FBG, $P<0.001$ ), serum low-density lipoprotein cholesterol (LDL-C, $P<0.001$ ), systolic blood pressure (SBP, $P<0.001$ ), diastolic blood pressure (DBP, $P<0.001$ ), and total cholesterol (TC, $P<0.001$ ) were also significantly different between cases and controls. However, triglyceride (TG, $P=0.412$ ) is no significant association between cases and controls in the north of China, and high-density lipoprotein cholesterol (HDL-C, $P=0.714$ ) is no significant association between cases and controls in the south of China.

Genotype distribution of the PVT1 rs10956390 and rs80177647 polymorphisms in both controls and $\mathrm{EH}$ patients were in agreement with the Hardy-Weinberg Equilibrium (Table 2). 
TABLE 1: General characteristics of the EH case and control population.

\begin{tabular}{lccccccccc}
\hline \multirow{2}{*}{ Characteristics } & \multicolumn{2}{c}{ The south of China } & \multicolumn{3}{c}{ The north of China } & \multicolumn{3}{c}{ Total } \\
& Control & EH & $P$ & Control & EH & $P$ & Control & EH & $P$ \\
\hline Gender & 294 & 261 & & 145 & 263 & & 439 & 524 \\
Male (\%) & $173(46.6 \%)$ & $157(60.2 \%)$ & & $75(51.7 \%)$ & $130(49.4 \%)$ & & $248(56.5 \%)$ & $287(54.8 \%)$ \\
Female (\%) & $121(53.4 \%)$ & $104(39.8 \%)$ & 0.754 & $70(48.3 \%)$ & $133(50.6 \%)$ & 0.658 & $191(43.5 \%)$ & $237(45.2 \%)$ & 0.593 \\
Age (years) & $52 \pm 8$ & $54 \pm 8.0$ & 0.098 & $53 \pm 9$ & $53 \pm 8$ & 0.844 & $53 \pm 8$ & $53 \pm 8$ & 0.223 \\
BMI (kg/m $\left.{ }^{2}\right)$ & $22.20 \pm 1.63$ & $24.76 \pm 2.71$ & $<0.001$ & $22.64 \pm 1.84$ & $24.94 \pm 3.34$ & $<0.001$ & $22.35 \pm 1.71$ & $24.85 \pm 3.04$ & $<0.001$ \\
SBP (mmHg) & $120 \pm 12$ & $136 \pm 20$ & $<0.001$ & $122 \pm 10$ & $138 \pm 19$ & $<0.001$ & $120 \pm 11$ & $137 \pm 19$ & $<0.001$ \\
DBP (mmHg) & $76 \pm 8$ & $85 \pm 13$ & $<0.001$ & $75 \pm 9$ & $80 \pm 13$ & $<0.001$ & $76 \pm 8$ & $83 \pm 13$ & $<0.001$ \\
Smoking history $(\%)$ & $38(12.9 \%)$ & $63(24.1 \%)$ & 0.001 & $20(13.8 \%)$ & $80(30.4 \%)$ & $<0.001$ & $58(13.2 \%)$ & $143(27.3 \%)$ & $<0.001$ \\
FBG, mmoL/L & $4.54 \pm 0.48$ & $5.4 \pm 1.17$ & $<0.001$ & $4.75 \pm 1.49$ & $5.5 \pm 0.87$ & $<0.001$ & $4.61 \pm 0.95$ & $5.45 \pm 1.03$ & $<0.001$ \\
TG, mmol/L & $1.30 \pm 0.46$ & $1.86 \pm 1.99$ & $<0.001$ & $1.36 \pm 0.43$ & $1.41 \pm 0.72$ & 0.412 & $1.32 \pm 0.45$ & $1.64 \pm 0.89$ & $<0.001$ \\
TC, mmol/L & $4.42 \pm 0.62$ & $4.83 \pm 0.92$ & $<0.001$ & $4.22 \pm 0.63$ & $4.57 \pm 0.97$ & $<0.001$ & $4.36 \pm 0.63$ & $4.70 \pm 0.95$ & $<0.001$ \\
HDL-C, mmol/L & $1.27 \pm 0.35$ & $1.28 \pm 0.41$ & 0.741 & $1.26 \pm 0.33$ & $1.36 \pm 0.52$ & 0.013 & $1.26 \pm 0.34$ & $1.32 \pm 0.47$ & 0.032 \\
LDL-C, mmol/L & $2.52 \pm 0.62$ & $2.74 \pm 0.81$ & $<0.001$ & $2.19 \pm 0.52$ & $2.41 \pm 0.75$ & $<0.001$ & $2.41 \pm 0.61$ & $2.58 \pm 0.80$ & $<0.001$ \\
\hline
\end{tabular}

EH: essential hypertension; BMI: body mass index; SBP: systolic blood pressure; DBP: diastolic blood pressure; FBG: fasting blood glucose; TC: total cholesterol; TG: triglyceride; HDL-C: high-density lipoprotein cholesterol; LDL-C: low-density lipoprotein cholesterol.

3.2. Association of PVT1 rs10956390 C>T and rs80177647 $T>A$ Polymorphisms and Risk for EH in the South of China. Table 3 shows the association between PVT1 polymorphisms and $\mathrm{EH}$ risk in the south of China. Logistic regression analysis showed that rs10956390 CT and TT genotypes were associated with decreased risk of $\mathrm{EH}$ (additive model: CT: odds ratio $(\mathrm{OR})=0.657,95 \%$ confidence interval $(\mathrm{CI})=0.445-0.968, P=0.034$; TT: $\mathrm{OR}=0.571,95 \%$ $\mathrm{CI}=0.355-0.918, P=0.021$; dominant model: $\mathrm{OR}=0.629$, 95\% CI $=0.437-0.906, P=0.013)$. However, no significant association was observed after adjustment for age, gender, BMI, smoking history, FBG, and dyslipidemia.

In addition, we also found that rs80177647 A allele was associated with increased risk of $\mathrm{EH}$ (additive model: TA: $\mathrm{OR}=1.855, \quad 95 \% \quad \mathrm{CI}=1.181-2.914, \quad P=0.007 ;$ recessive model: $\mathrm{OR}=1.842,95 \% \mathrm{CI}=1.188-2.855, P=0.006)$. Moreover, the adjusted result was also significant (additive model: TA: $\mathrm{OR}=1.950,95 \% \mathrm{CI}=1.023-3.781, P=0.042$; dominant model: $\mathrm{OR}=1.956,95 \% \mathrm{CI}=1.041-3.676, P=0.037)$.

3.3. Association of PVT1 rs10956390 C>T and rs80177647 T>A Polymorphisms and Risk for EH in the North of China. Logistic regression analyses revealed that $\mathrm{EH}$ risk was decreased significantly in carriers of $\mathrm{T}$ allele of rs10956390 polymorphism than CC genotype (additive model: $\mathrm{CT}$ : $\mathrm{OR}=0.525,95 \% \mathrm{CI}=0.321-0.860, P=0.010$; TT: $\mathrm{OR}=0.402,95 \% \mathrm{CI}=0.228-0.712, P=0.002$; dominant model: $\mathrm{OR}=0.480,95 \% \mathrm{CI}=0.303-0.762, P=0.002$; recessive model: $\mathrm{OR}=0.595,95 \% \mathrm{CI}=0.370-0.957, P=0.032$ ) as shown in Table 4. And after adjustment, the association was still significant (additive model: CT: OR $=0.409,95 \%$ $\mathrm{CI}=0.221-0.757, \quad P=0.004 ; \quad \mathrm{TT}: \quad \mathrm{OR}=0.341,95 \% \quad \mathrm{CI}=$ 0.168-0.693, $P=0.003$; dominant model: $\mathrm{OR}=0.384,95 \%$ $\mathrm{CI}=0.216-0.684, P=0.001)$.

While compared with TT genotype of rs80177647, A allele carriers have increased $\mathrm{EH}$ risk (additive model: TA:
TABLE 2: Test results of Hardy-Weinberg Equilibrium among all PVT1 gene sites.

\begin{tabular}{lccccccccc}
\hline \multirow{2}{*}{ Site } & \multicolumn{3}{c}{ South of China } & \multicolumn{4}{c}{ North of China } \\
& \multicolumn{2}{c}{ Control } & \multicolumn{2}{c}{ EH } & \multicolumn{3}{c}{ Control } & \multicolumn{2}{c}{ EH } \\
& $\chi^{2}$ & $P$ & $\chi^{2}$ & $P$ & $\chi^{2}$ & $P$ & $\chi^{2}$ & $P$ \\
\hline rs10956390 & 0.06 & 0.81 & 0.78 & 0.38 & 0.05 & 0.83 & 3.09 & 0.08 \\
rs80177647 & 1.30 & 0.25 & 0.001 & 0.96 & 1.53 & 0.22 & 0.11 & 0.74 \\
\hline
\end{tabular}

$\mathrm{OR}=2.119, \quad 95 \% \quad \mathrm{CI}=1.210-3.711, \quad P=0.009 ;$ dominant model: $\mathrm{OR}=2.100,95 \% \mathrm{CI}=1.226-3.596, P=0.007)$. However, no significant association was observed after adjustment.

3.4. Association of PVT1 Polymorphisms and Risk for EH in China. Then, we aggregated and analyzed all the data from the two centers in southern and northern China. We found that the polymorphisms of the two SNPs of PVT1 were associated with the risk of $\mathrm{EH}$. Logistic regression analysis showed that rs10956390 CT and TT genotypes were associated with decreased risk of $\mathrm{EH}$ (additive model: $\mathrm{CT}$ : $\mathrm{OR}=0.596,95 \%$ $\mathrm{CI}=0.442-0.804, P=0.001 ; \mathrm{TT}: \mathrm{OR}=0.502,95 \% \mathrm{CI}=0.350$ -0.718, $P<0.001$; dominant model: $\mathrm{OR}=0.564,95 \% \mathrm{CI}=$ 0.426-0.747, $P<0.001$; recessive model: $\mathrm{OR}=0.688,95 \% \mathrm{CI}$ $=0.506-0.936, P=0.017$, Table 5). And after adjustment, the association was still significant (additive model: CT: OR $=0.488,95 \% \mathrm{CI}=0.326-0.729, P<0.001 ; \mathrm{TT}: \mathrm{OR}=0.460$, 95\% CI $=0.286-0.740, P=0.001$; dominant model: $\mathrm{OR}=$ $0.478,95 \% \mathrm{CI}=0.328-0.698, P<0.001)$.

Rs80177647 has similar results. The TA genotype and the dominant model showed stronger relations with higher EH risk $(P<0.001, \mathrm{OR}=1.993,95 \% \mathrm{CI}=1.410-2.816 ; P<$ $0.001, \quad \mathrm{OR}=1.986, \quad 95 \% \mathrm{CI}=1.422-2.774$, respectively). When adjusted for $\mathrm{EH}$ risk factors, including age, gender, BMI, smoking history, FBG, and dyslipidemia, the significant association between PVT1 rs80177647 was also 
TABLE 3: Association between lncRNA PVT1 polymorphisms and EH risk in the south of China.

\begin{tabular}{|c|c|c|c|c|c|c|c|}
\hline Models & Genotypes & $\begin{array}{c}\text { Control, } n=294 \\
(\%)\end{array}$ & $\begin{array}{c}\mathrm{EH}, n=261 \\
(\%)\end{array}$ & Unadjusted OR (95\% CI) & $P$ value & ${ }^{*}$ Adjusted OR (95\% CI) & ${ }^{*}$ Adjusted $P$ value \\
\hline \multicolumn{8}{|l|}{ rs10956390 } \\
\hline \multirow{3}{*}{ Additive } & $\mathrm{CC}$ & $75(25.5)$ & $92(35.2)$ & 1.00 (reference) & & 1.00 (reference) & \\
\hline & $\mathrm{CT}$ & $149(50.7)$ & $120(46.0)$ & $0.657(0.445-0.968)$ & 0.034 & $0.610(0.340-1.094)$ & 0.097 \\
\hline & $\mathrm{TT}$ & $70(23.8)$ & $49(18.8)$ & $0.571(0.355-0.918)$ & 0.021 & $0.657(0.319-1.354)$ & 0.254 \\
\hline \multirow{2}{*}{ Dominant } & $\mathrm{CC}$ & $75(25.5)$ & $92(35.2)$ & 1.00 (reference) & & 1.00 (reference) & \\
\hline & $\mathrm{CT} / \mathrm{TT}$ & $119(74.5)$ & $169(64.8)$ & $0.629(0.437-0.906)$ & 0.013 & $0.623(0.357-1.085)$ & 0.094 \\
\hline \multirow{2}{*}{ Recessive } & $\mathrm{CC} / \mathrm{CT}$ & $224(76.2)$ & $212(81.2)$ & 1.00 (reference) & & 1.00 (reference) & \\
\hline & $\mathrm{TT}$ & $70(23.8)$ & $49(18.8)$ & $0.740(0.491-1.115)$ & 0.150 & $0.911(0.494-1.680)$ & 0.766 \\
\hline \multicolumn{8}{|l|}{ rs80177647 } \\
\hline \multirow{3}{*}{ Additive } & $\mathrm{TT}$ & $253(86.1)$ & $201(77.0)$ & 1.00 (reference) & & 1.00 (reference) & \\
\hline & TA & $38(12.9)$ & $56(21.5)$ & $1.855(1.181-2.914)$ & 0.007 & $1.950(1.023-3.718)$ & 0.042 \\
\hline & AA & $3(1.0)$ & $4(1.5)$ & $1.678(0.371-7.585)$ & 0.501 & $2.063(0.183-23.305)$ & 0.558 \\
\hline \multirow{2}{*}{ Dominant } & $\mathrm{TT}$ & $253(86.1)$ & $201(77.0)$ & 1.00 (reference) & & 1.00 (reference) & \\
\hline & TA/AA & $41(13.9)$ & $60(23.0)$ & $1.842(1.188-2.855)$ & 0.006 & $1.956(1.041-3.676)$ & 0.037 \\
\hline \multirow{2}{*}{ Recessive } & TT/TA & $291(99.0)$ & $257(98.5)$ & 1.00 (reference) & & 1.00 (reference) & \\
\hline & AA & $3(1.0)$ & $4(1.5)$ & $1.510(0.335-6.809)$ & 0.592 & $1.843(0.163-20.785)$ & 0.621 \\
\hline
\end{tabular}

OR: odd ratio; CI: confidence interval; EH: essential hypertension. *Adjusted for age, gender, BMI, smoking history, FBG, and dyslipidemia.

TABLE 4: Association between lncRNA PVT1 polymorphisms and EH risk in the north of China.

\begin{tabular}{|c|c|c|c|c|c|c|c|}
\hline Models & Genotypes & $\begin{array}{c}\text { Control, } n=145 \\
(\%)\end{array}$ & $\begin{array}{c}\mathrm{EH}, n=263 \\
(\%)\end{array}$ & Unadjusted OR (95\% CI) & $P$ value & ${ }^{*}$ Adjusted OR (95\% CI) & ${ }^{*}$ Adjusted $P$ value \\
\hline \multicolumn{8}{|l|}{ rs10956390 } \\
\hline \multirow{3}{*}{ Additive } & $\mathrm{CC}$ & $33(22.8)$ & $100(38.0)$ & 1.00 (reference) & & 1.00 (reference) & \\
\hline & CT & $71(48.3)$ & $113(43.0)$ & $0.525(0.321-0.860)$ & 0.010 & $0.409(0.221-0.757)$ & 0.004 \\
\hline & TT & $41(28.3)$ & $50(19.0)$ & $0.402(0.228-0.712)$ & 0.002 & $0.341(0.168-0.693)$ & 0.003 \\
\hline \multirow{2}{*}{ Dominant } & $\mathrm{CC}$ & $33(22.8)$ & $100(38.0)$ & 1.00 (reference) & & 1.00 (reference) & \\
\hline & $\mathrm{CT} / \mathrm{TT}$ & $112(77.2)$ & $163(62.0)$ & $0.480(0.303-0.762)$ & 0.002 & $0.384(0.216-0.684)$ & 0.001 \\
\hline \multirow{2}{*}{ Recessive } & $\mathrm{CC} / \mathrm{CT}$ & $104(71.7)$ & $223(81.0)$ & 1.00 (reference) & & 1.00 (reference) & \\
\hline & $\mathrm{TT}$ & $41(28.3)$ & $50(19.0)$ & $0.595(0.370-0.957)$ & 0.032 & $0.598(0.333-1.072)$ & 0.084 \\
\hline \multicolumn{8}{|l|}{ rs80177647 } \\
\hline \multirow{3}{*}{ Additive } & $\mathrm{TT}$ & $124(85.5)$ & $194(73.8)$ & 1.00 (reference) & & 1.00 (reference) & \\
\hline & TA & $19(13.1)$ & $63(24.0)$ & $2.119(1.210-3.711)$ & 0.009 & $1.535(0.802-2.938)$ & 0.196 \\
\hline & AA & $2(1.4)$ & $6(2.3)$ & $1.918(0.381-9.652)$ & 0.430 & $0.734(0.109-4.944)$ & 0.751 \\
\hline \multirow{2}{*}{ Dominant } & $\mathrm{TT}$ & $124(85.5)$ & $194(73.8)$ & 1.00 (reference) & & 1.00 (reference) & \\
\hline & TA/AA & $21(14.5)$ & $69(26.3)$ & $2.100(1.226-3.596)$ & 0.007 & $1.449(0.775-2.710)$ & 0.246 \\
\hline \multirow{2}{*}{ Recessive } & TT/TA & $143(98.6)$ & $257(97.7)$ & 1.00 (reference) & & 1.00 (reference) & \\
\hline & $\mathrm{AA}$ & $2(1.4)$ & $6(2.3)$ & $1.669(0.333-8.379)$ & 0.534 & $0.664(0.098-4.483)$ & 0.674 \\
\hline
\end{tabular}

OR: odd ratio; CI: confidence interval; EH: essential hypertension. *Adjusted for age, gender, BMI, smoking history, FBG, and dyslipidemia.

observed (additive model: TA: $\mathrm{OR}=1.768,95 \% \mathrm{CI}=1.144$ -2.731, $P=010$; dominant model: $\mathrm{OR}=1.723,95 \% \mathrm{CI}=$ $1.128-2.631, P=0.012)$.

3.5. Influence of PVT1 rs10956390 C>T and rs80177647 $T>A$ Polymorphisms of Lipid Levels in Subjects. Lipid levels, such as triglyceride, total cholesterol, high-density lipoprotein, and low-density lipoprotein were determined in $963 \mathrm{EH}$ patients and normal controls. Moreover, the effects of rs10956390 and rs80177647 of PVT1 genotypes on lipid levels were analyzed. In controls, TG levels in CT and TT genotypes were significantly lower than those in subjects for rs10956390 CC genotype $\left({ }^{*} P<0.05\right.$, Figure 1(a)). And in EH patients, TG levels in TT genotype were lower than CT genotype $(\# P<0.05$, Figure 1(a)). More interestingly, we found that HDL-C levels in CT and TT genotypes were higher than CC genotype in control and $\mathrm{EH}$ patients, respectively $\left({ }^{*} P<0.05,{ }^{* *} P<0.01\right.$, Figure $1(\mathrm{c})$ ). What is more, the levels of LDL-C were lower in 
TABle 5: Association between lncRNA PVT1 polymorphisms and EH risk in China.

\begin{tabular}{|c|c|c|c|c|c|c|c|}
\hline Models & Genotypes & $\begin{array}{c}\text { Control, } n=439 \\
(\%)\end{array}$ & $\begin{array}{c}\mathrm{EH}, n=524 \\
(\%)\end{array}$ & Unadjusted OR (95\% CI) & $P$ value & ${ }^{*}$ Adjusted OR (95\% CI) & ${ }^{*}$ Adjusted $P$ value \\
\hline \multicolumn{8}{|l|}{ rs10956390 } \\
\hline \multirow{3}{*}{ Additive } & $\mathrm{CC}$ & $108(24.6)$ & $192(36.6)$ & 1.00 (reference) & & 1.00 (reference) & \\
\hline & $\mathrm{CT}$ & $220(50.1)$ & $233(44.5)$ & $0.596(0.442-0.804)$ & 0.001 & $0.488(0.326-0.729)$ & $<0.001$ \\
\hline & $\mathrm{TT}$ & $111(25.3)$ & 99 (18.9) & $0.502(0.350-0.718)$ & $<0.001$ & $0.460(0.286-0.740)$ & 0.001 \\
\hline \multirow{2}{*}{ Dominant } & $\mathrm{CC}$ & $108(24.6)$ & $192(36.6)$ & 1.00 (reference) & & 1.00 (reference) & \\
\hline & $\mathrm{CT} / \mathrm{TT}$ & $331(75.4)$ & $332(63.4)$ & $0.564(0.426-0.747)$ & $<0.001$ & $0.478(0.328-0.698)$ & $<0.001$ \\
\hline \multirow{2}{*}{ Recessive } & $\mathrm{CC} / \mathrm{CT}$ & $328(74.7)$ & $425(81.1)$ & 1.00 (reference) & & 1.00 (reference) & \\
\hline & $\mathrm{TT}$ & $111(25.3)$ & $99(18.9)$ & $0.688(0.506-0.936)$ & 0.017 & $0.721(0.483-1.077)$ & 0.110 \\
\hline \multicolumn{8}{|l|}{ rs80177647 } \\
\hline \multirow{3}{*}{ Additive } & $\mathrm{TT}$ & $377(85.9)$ & $395(75.4)$ & 1.00 (reference) & & 1.00 (reference) & \\
\hline & TA & $57(13.0)$ & $119(22.7)$ & $1.993(1.410-2.816)$ & $<0.001$ & $1.768(1.144-2.731)$ & 0.010 \\
\hline & $\mathrm{AA}$ & $5(1.1)$ & $10(1.9)$ & $1.909(0.646-5.636)$ & 0.242 & $1.170(0.250-5.40)$ & 0.842 \\
\hline \multirow{2}{*}{ Dominant } & $\mathrm{TT}$ & 377 (85.9) & $395(75.4)$ & 1.00 (reference) & & 1.00 (reference) & \\
\hline & TA/AA & $62(14.1)$ & $129(24.6)$ & $1.986(1.422-2.774)$ & $<0.001$ & $1.723(1.128-2.631)$ & 0.012 \\
\hline \multirow{2}{*}{ Recessive } & TT/TA & 434 (98.9) & $514(98.1)$ & 1.00 (reference) & & 1.00 (reference) & \\
\hline & $\mathrm{AA}$ & $5(1.1)$ & $10(1.9)$ & $1.689(0.573-4.978)$ & 0.342 & $1.045(0.221-4.927)$ & 0.956 \\
\hline
\end{tabular}

OR: odd ratio; CI: confidence interval; EH: essential hypertension. *Adjusted for age, gender, BMI, smoking history, FBG, and dyslipidemia.
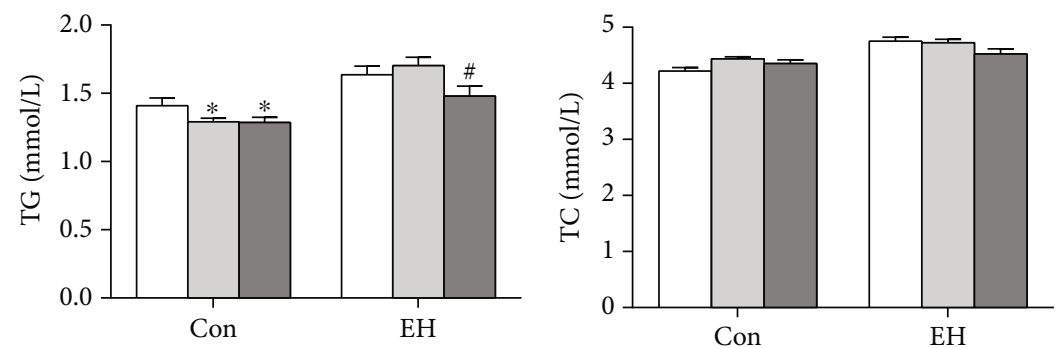

$\square$ CC
$\square$ CT
$\square \mathrm{TT}$

(a)

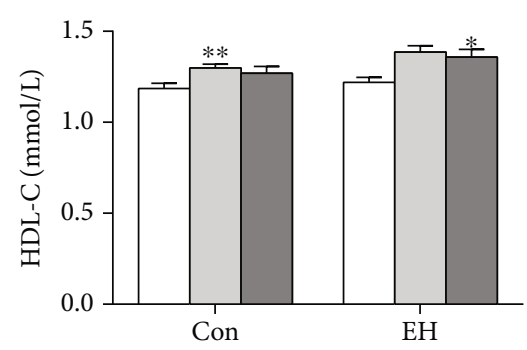

$\square \mathrm{CC}$

$\square \mathrm{CT}$

$\square \mathrm{TT}$
$\square \mathrm{CC}$
$\square$ CT

$\square \mathrm{TT}$

(b)

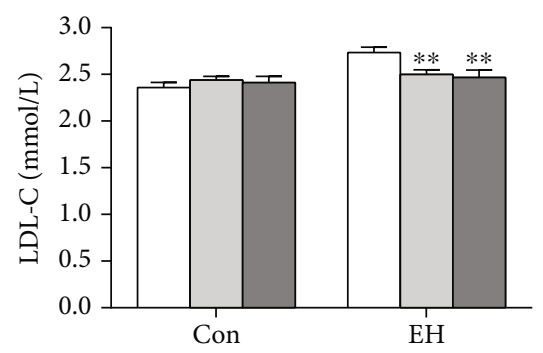

$\square \mathrm{CC}$

$\square \mathrm{CT}$

$\square \mathrm{TT}$

(c)

(d)

Figure 1: Influence of PVT1 rs10956390 polymorphism on lipid level of subjects: (a) TG; (b) TC; (c) HDL-C; (d) LDL-C. (Data are expressed as the mean \pm SEM. Control: CC, $n=108$, CT, $n=220$, TT, $n=111$; EH: CC, $n=192, \mathrm{CT}, n=233, \mathrm{TT}, n=99 .{ }^{*} P<0.05,{ }^{* *} P$ $<0.01$, as compared with CC genotype in interclass; ${ }^{\#} P<0.05$, as compared with CT genotype in interclass). 

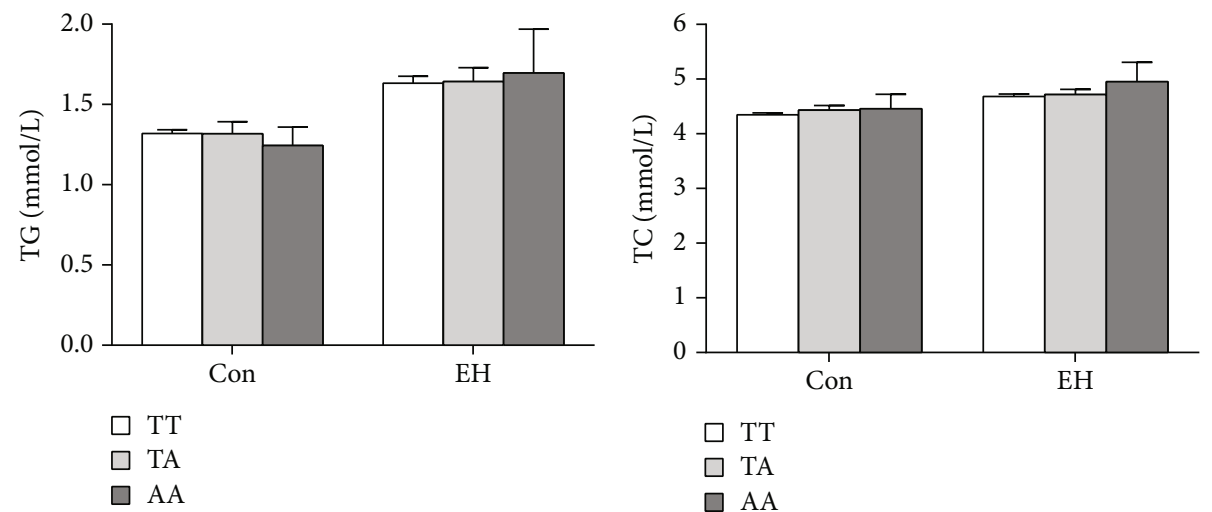

$$
\begin{aligned}
& \square \text { TT } \\
& \square \text { TA } \\
& \square \text { AA }
\end{aligned}
$$

(a)
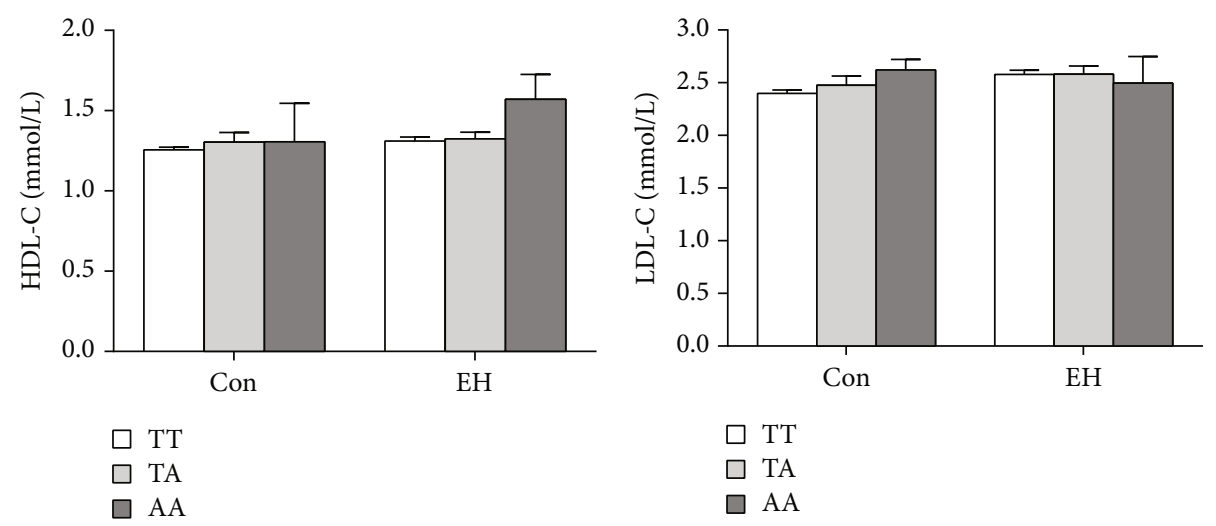

(c)

(d)

FIGURE 2: Influence of PVT1 rs80177647 polymorphism on lipid level of subjects: (a) TG; (b) TC; (c) HDL-C; (d) LDL-C. (Data are expressed as the mean \pm SEM. Control: TT, $n=377$, TA, $n=57$, AA, $n=5$; EH: TT, $n=395$, TA, $n=119$, AA, $n=10$.).

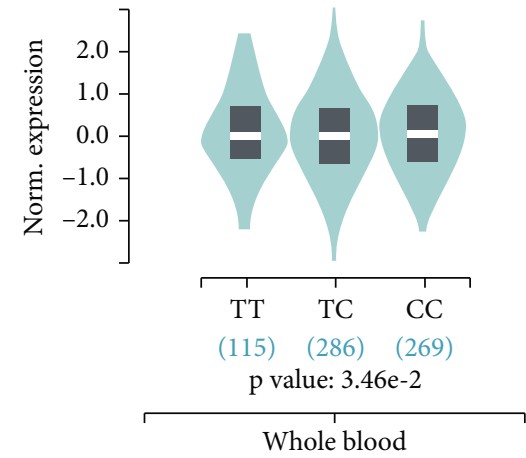

(a)

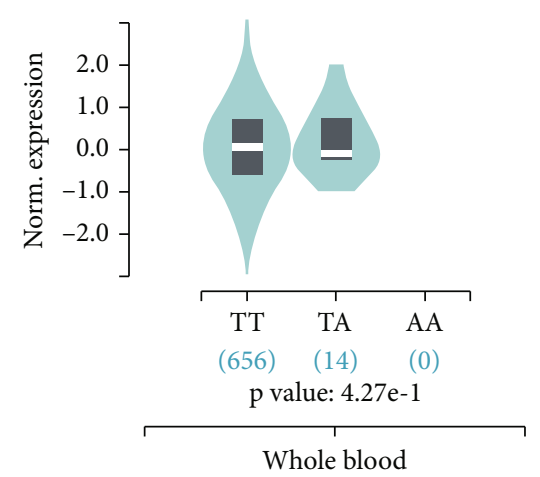

(b)

FIGURE 3: Effect of PVT1 mRNA expression in whole blood from healthy normal donors: (a) rs10956390; (b) rs80177647.

$\mathrm{CT}$ and TT genotypes in EH patients $\left({ }^{* *} P<0.01\right.$, compared with CC genotype, Figure 1(c)). However, by comparing the correlation between rs80177647 and lipid level in different subjects, we did not find significant differences between lipid levels and genotypes (Figure 2).

3.6. Association of PVT1 rs10956390 and rs80177647 Polymorphisms with PVT1 Expression. The Genotype-Tissue Expression (GTEx) project is one of the most widely used resources for studying the relationship between genetic varia- tion and gene expression. This dataset contains genotype data from 838 postmortem donors and 17,382 RNA-seq samples across 54 tissue sites and 2 cell lines. We accessed the database to determine whether rs 10956390 and rs80177647 polymorphisms were associated with PVT1 expression in multitissue through the GTEx eQTL Dashboard (https://www.gtexportal .org/home/eqtlDashboardPage). As shown in Figure 3(a), rs10956390 T allele was associated with decreased PVT1 mRNA in whole blood from 670 healthy donors (the relative expression median: TT: -0.04297 , TC: -0.05181 , CC: 0.02802 , 
$P=0.0346)$. But there was no significant difference between rs80177647 polymorphism and PVT1 mRNA expression (Figure 3(b), $P=0.427$ ).

\section{Discussion}

This study reveals, for the first time, there is an association between rs80177647 and rs10956390 gene polymorphisms of PVT1 and the risk of essential hypertension in two populations in southern and northern China. Our results demonstrated that EH risk was significantly decreased in carriers of $\mathrm{T}$ allele of rs 10956390 polymorphism than those with the CC genotype. In addition, we also found that $\mathrm{EH}$ risk was significantly increased in carriers of A allele of rs80177647 polymorphism than those with TT.

The majority of studies in the past have focused on the relationship between PVT1 and the occurrence and development of various cancers. It is reported that PVT1 is typically upregulated in many types of cancer samples [20]. Some of those have found that PVT1 was involved in the regulation of angiogenesis in tumor tissues. Ma et al. showed that PVT1 overexpression promoted the proliferation, migration, and angiogenesis of glioma vascular endothelial cells [21]. However, a few recent studies have implied that PVT1 was involved in cardiovascular diseases. For instance, PVT1 acted as a sponge for miR-128-3p to facilitate Sp1 expression, resulting in activating the TGF- $\beta 1 /$ Smad signaling pathway and regulating the development of atrial fibrosis [22]. Zhang et al. also found that PVT1 expression was significantly upregulated in abdominal aortic tissues from AAA patients, and knockdown PVT1 in AngII-induced AAA murine model suppresses VSMC apoptosis, ECM disruption, and serum proinflammatory cytokine level [23]. Strikingly, Quan et al. revealed that PVT1 was proved to be an independent risk factor for coronary atherosclerosis disease [24]. Thus, all those researches indicated the role of PVT1 involved in diseases is related to angiogenesis and endothelial dysfunction.

Previous studies in the association between PVT1 polymorphisms and diseases were only reported in the field of cancer and kidney disease. Our present data found two new SNP loci, rs10956390 and rs80177647, which polymorphisms were related to the risk of $\mathrm{EH}$ in Chinese population. However, the underlying mechanism is still unclear. IncRNAs play crucial roles in many human diseases; their structure and subcellular localization determine their functions [25]. A large amount of evidence has been demonstrated that the causality relationship between higher grade disequilibrium in endothelial homeostasis and diabetes or hypertension. The risky factors from vascular endothelial cell including reduced plasma nitric oxide level, suppressed L-arginine/endothelial nitric oxide synthase (eNOS) pathway, generation of reactive oxygen species (ROS), and enhanced leukocyte adhesion to the vascular wall may cooperatively contribute to the pathogenesis and progress of EH [26]. In our study, there were significant differences in TG, LDL-C, and HDL-C levels among rs10956390. Similarly, Wang et al. revealed that APOE-E3 homozygote and APOE$\mathrm{E} 4$ allele were related to elevated triglycerides level; in addition, APOE-E2 allele was correlated with increased serum UA level in patients with hypertension or coronary heart disease [27]. Pan et al. found that ACE2 rs4646188 and rs879922 were associated with increased LDL-C level, while rs2106809 and rs4646188 were associated with hypertriglyceridemia [28]. The evidence we mentioned above demonstrates that different alleles in one gene may affect blood lipids differently. Most importantly, numerous studies have been revealed that there is a positive correlation between dyslipidemia and hypertension $[29,30]$. A newly published study found that PVT1 was upregulated in the adipose tissue of obese mice and accelerated lipid accumulation by increasing the expression of peroxisome proliferator-activated receptor $\gamma$, CCAAT/enhancer-binding protein $\alpha$, and adipocyte protein 2 . In addition, PVT1 promoted fatty acid synthesis and inhibited fatty acid oxidation [17]. Based on all those studies and our data, we speculate that PVT1 may affect the risk of EH by regulating human blood lipid levels.

Several limitations of this study should be considered. Firstly, a limited number of samples were chosen in this study. Secondly, only the association between the PVT1 polymorphisms of the two loci, the risks of $\mathrm{EH}$ and plasma lipid levels have been investigated. However, the precise mechanism of how PVT1 influences lipid level in vivo or in vitro has not been studied. Further mechanism studies and larger populationbased prospective studies are required.

\section{Conclusion}

In summary, our study demonstrates firstly that the PVT1 rs10956390 and rs80177647 polymorphisms are associated with the risk of $\mathrm{EH}$ in Chinese population. We speculate that the rs 10956390 polymorphism is responsible for dyslipidemia. PVT1 is a potential biomarker and target for therapeutic strategies in EH. Further investigations in larger cohorts are needed to confirm our findings. More functional experiments are also required to illuminate the function role of rs10956390 and rs80177647 polymorphisms.

\section{Data Availability}

The research data used to support the findings of this study are available from the corresponding author upon request.

\section{Conflicts of Interest}

The authors declare that they have no competing interests.

\section{Authors' Contributions}

DBK and RL conceived and designed the experiments. XY, YC, and MLX performed the experiments. MLZ, ZSY, and YLX analyzed the data. RL and XY contributed reagents/ materials/analysis tools. DBK wrote the paper. All authors read and approved the final manuscript.

\section{Acknowledgments}

This work was supported by research grants of the National Natural Science Foundation of China (No. 81803767), the Hunan Provincial Natural Science Foundation of China 
(No. 2021JJ40617), the Hospital Pharmacy Research Funds of Hunan Pharmaceutical Association (2020YXH005), the Science and Technology Project of Hengyang City (202010031549), the Self-financing Scientific Research Project of Guangxi Health Commission (grant number Z20201003), and the Hospital Management Research and Reform Project of University of South China (2019YYGL02).

\section{References}

[1] "Global, regional, and national age-sex specific mortality for 264 causes of death, 1980-2016: a systematic analysis for the Global Burden of Disease Study 2016," Lancet, vol. 390, no. 10100, pp. 1151-1210, 2017.

[2] D. Colomba, G. Duro, S. Corrao et al., "Endothelial nitric oxide synthase gene polymorphisms and cardiovascular damage in hypertensive subjects: an Italian case-control study," Immunity \& Ageing, vol. 5, no. 1, p. 4, 2008.

[3] P. J. Batista and H. Y. Chang, "Long noncoding RNAs: cellular address codes in development and disease," Cell, vol. 152, no. 6, pp. 1298-1307, 2013.

[4] M. Zeng, C. Lu, F. Zhang et al., "SDLDA: lncRNA-disease association prediction based on singular value decomposition and deep learning," Methods, vol. 179, pp. 73-80, 2020.

[5] C. Y. Liu, Y. H. Zhang, R. B. Li et al., "LncRNA CAIF inhibits autophagy and attenuates myocardial infarction by blocking p53-mediated myocardin transcription," Nature Communications, vol. 9, no. 1, p. 29, 2018.

[6] L. Shi, C. Tian, L. Sun, F. Cao, and Z. Meng, "The lncRNA TUG1/miR-145-5p/FGF10 regulates proliferation and migration in VSMCs of hypertension," Biochemical and Biophysical Research Communications, vol. 501, no. 3, pp. 688-695, 2018.

[7] M. Xiao, Y. Feng, C. Liu, and Z. Zhang, "Prognostic values of long noncoding RNA PVT1 in various carcinomas: an updated systematic review and meta-analysis," Cell Proliferation, vol. 51, no. 6, article e12519, 2018.

[8] L. You, H. Wang, G. Yang et al., "Gemcitabine exhibits a suppressive effect on pancreatic cancer cell growth by regulating processing of PVT1 to miR1207," Molecular Oncology, vol. 12, no. 12, pp. 2147-2164, 2018.

[9] Z. Chang, J. Cui, and Y. Song, "Long noncoding RNA PVT1 promotes EMT via mediating microRNA-186 targeting of Twist1 in prostate cancer," Gene, vol. 654, pp. 36-42, 2018.

[10] Y. Wang, X. Li, W. Liu et al., "MicroRNA-1205, encoded on chromosome 8q24, targets EGLN3 to induce cell growth and contributes to risk of castration-resistant prostate cancer," Oncogene, vol. 38, no. 24, pp. 4820-4834, 2019.

[11] Z. Tian, S. Cao, C. Li et al., "LncRNA PVT1 regulates growth, migration, and invasion of bladder cancer by miR-31/CDK1," Journal of Cellular Physiology, vol. 234, no. 4, pp. 4799-4811, 2019.

[12] L. Yang, X. Peng, H. Jin, and J. Liu, "Long non-coding RNA PVT1 promotes autophagy as ceRNA to target ATG3 by sponging microRNA-365 in hepatocellular carcinoma," Gene, vol. 697, pp. 94-102, 2019.

[13] J. Zhao, P. du, P. Cui et al., "LncRNA PVT1 promotes angiogenesis via activating the STAT3/VEGFA axis in gastric cancer," Oncogene, vol. 37, no. 30, pp. 4094-4109, 2018.

[14] J. Zheng, L. Hu, J. Cheng et al., "IncRNA PVT1 promotes the angiogenesis of vascular endothelial cell by targeting miR26b to activate CTGF/ANGPT2," International Journal of Molecular Medicine, vol. 42, no. 1, pp. 489-496, 2018.

[15] B. Sun, M. Meng, J. Wei, and S. Wang, "Long noncoding RNA PVT1 contributes to vascular endothelial cell proliferation via inhibition of miR-190a-5p in diagnostic biomarker evaluation of chronic heart failure," Experimental and Therapeutic Medicine, vol. 19, no. 5, pp. 3348-3354, 2020.

[16] J. Guo, J. Li, J. Zhang et al., "LncRNA PVT1 knockdown alleviated ox-LDL-induced vascular endothelial cell injury and atherosclerosis by miR-153-3p/GRB2 axis via ERK/p38 pathway," Nutrition, Metabolism, and Cardiovascular Diseases, vol. 31, no. 12, pp. 3508-3521, 2021.

[17] L. Zhang, D. Zhang, Z. Y. Qin, J. Li, and Z. Y. Shen, "The role and possible mechanism of long noncoding RNA PVT1 in modulating 3T3-L1 preadipocyte proliferation and differentiation," IUBMB Life, vol. 72, no. 7, pp. 1460-1467, 2020.

[18] G. Seravalle and G. Grassi, "Obesity and hypertension," Pharmacological Research, vol. 122, pp. 1-7, 2017.

[19] Y. Lu, W. Yuan, L. Wang et al., "Contribution of lncRNA CASC8, CASC11, and PVT1 genetic variants to the susceptibility of coronary heart disease," Journal of Cardiovascular Pharmacology, vol. 77, no. 6, pp. 756-766, 2021.

[20] D. Guo, Y. Wang, K. Ren, and X. Han, "Knockdown of LncRNA PVT1 inhibits tumorigenesis in non-small-cell lung cancer by regulating miR-497 expression," Experimental Cell Research, vol. 362, no. 1, pp. 172-179, 2018.

[21] Y. Ma, P. Wang, Y. Xue et al., "PVT1 affects growth of glioma microvascular endothelial cells by negatively regulating miR186," Tumour Biology, vol. 39, no. 3, p. 1010428317694326 , 2017.

[22] F. Cao, Z. Li, W. M. Ding, L. Yan, and Q. Y. Zhao, "LncRNA PVT1 regulates atrial fibrosis via miR-128-3p-SP1-TGF- $\beta 1$ Smad axis in atrial fibrillation," Molecular Medicine, vol. 25, no. 1, p. 7, 2019.

[23] Z. Zhang, G. Zou, X. Chen et al., "Knockdown of lncRNA PVT1 inhibits vascular smooth muscle cell apoptosis and extracellular matrix disruption in a murine abdominal aortic aneurysm model," Molecules and Cells, vol. 42, no. 3, pp. 218-227, 2019.

[24] W. Quan, P. F. Hu, X. Zhao, C. G. Lianhua, and B. R. Batu, "Expression level of lncRNA PVT1 in serum of patients with coronary atherosclerosis disease and its clinical significance," European Review for Medical and Pharmacological Sciences, vol. 24, no. 11, pp. 6333-6337, 2020.

[25] M. Zeng, Y. Wu, C. Lu, F. Zhang, F. X. Wu, and M. Li, "DeepLncLoc: a deep learning framework for long noncoding RNA subcellular localization prediction based on subsequence embedding," Briefings in Bioinformatics, 2021.

[26] H. N. Zhang, Q. Q. Xu, A. Thakur et al., "Endothelial dysfunction in diabetes and hypertension: role of microRNAs and long non-coding RNAs," Life Sciences, vol. 213, pp. 258-268, 2018.

[27] C. Wang, W. Yan, H. Wang, J. Zhu, and H. Chen, "APOE polymorphism is associated with blood lipid and serum uric acid metabolism in hypertension or coronary heart disease in a Chinese population," Pharmacogenomics, vol. 20, no. 14, pp. 1021-1031, 2019.

[28] Y. Pan, T. Wang, Y. Li et al., "Association of ACE2 polymorphisms with susceptibility to essential hypertension and dyslipidemia in Xinjiang, China," Lipids in Health and Disease, vol. 17 , no. 1, p. 241, 2018. 
[29] T. Otsuka, H. Takada, Y. Nishiyama et al., "Dyslipidemia and the risk of developing hypertension in a working-age male population," Journal of the American Heart Association, vol. 5, no. 3, article e003053, 2016.

[30] C. Borghi, F. Rodriguez-Artalejo, G. de Backer et al., "The association between blood pressure and lipid levels in Europe," Journal of Hypertension, vol. 34, no. 11, pp. 2155-2163, 2016. 